\title{
CARCINOMA OF THE BRONCHUS PRESENTING AS A CEREBELLAR NEUROPATHY
}

\author{
M. H. Oelbaum, B.Sc., M.D., M.R.C.P. \\ Consultant Physician \\ R. Statham, M.B., Ch.B. \\ Senior House Officer \\ Crumpsall Hospital, Manchester 8
}

THE protean manifestations of carcinoma of the bronchus are becoming increasingly recognized, and amongst the most interesting are the various neuropathies which may be encountered. These neurological syndromes, occurring independently of secondary deposits, are classified by Brain and Henson (1958) as cortical cerebellar degeneration, mixed forms, sensory neuropathy, peripheral neuropathy (sensorimotor) and neuromuscular.

An account follows of a patient presenting with a purely cerebellar picture with no pulmonary symptoms or signs, and in whom a bronchial carcinoma was demonstrated only at post-mortem examination.

\section{Case Report}

The patient, a woman aged 62, was admitted to Crumpsall Hospital on November 24, 1959, with a one-week's history of vague pains around both hips and down both legs. During this time increasing unsteadiness in walking had developed to such an extent that the patient was unable to stand unaided. For several days there had been some weakness of the left arm and hand with paræsthesiæ which also affected the left leg. Some slight blurring of vision and occasional diplopia had been noted. There had been no headache, vomiting, cough or weight loss. There was no relevant past or family history.

On examination the only abnormalities were in the central nervous system. There was nystagmus on looking to the left and marked signs of cerebellar ataxia chiefly affecting the left arm and leg. Speech was normal. The optic fundi and pupils showed no abnormality. All the tendon reflexes were generally depressed, the plantar responses were flexor and there was no cutaneous sensory loss.

Investigations. $\mathrm{Hb}$. was $14.2 \mathrm{~g} . / 100 \mathrm{ml}$., white cells 5,400 c.mm., ESR $19 \mathrm{~mm}$. in I hour. Chest and skull X-rays were normal. Examination of the cerebrospinal fluid showed only 2 lymphocytes/c.mm., the protein content was $57 \mathrm{mg}$./100 ml., the Pandy test gave a faint opalescence and the Lange was negative. The EEG was normal.

Within two weeks of admission she deteriorated rapidly. Gross dysarthria developed and, in additionir dysphagia. The ataxia became generalized but stif greater on the left side, and there was now nystagmus to both sides. There was poor palatal movement? worse on the left. No pyramidal disturbance was found at this stage, but six weeks after admission both plantaf responses were extensor. Her general condition gradually worsened and her mental state varied betweem euphoria and resentful depression. She becane increasingly apathetic and eventually incontinent ans died on June $21,1960$.

Post-mortem Examination.-Abnormalities restricted to the lungs and central nervous system Bronchopneumonia was present at both lung bases A small mass of white growth $3.5 \mathrm{~cm} . \times 2.5 \mathrm{~cm}$ surrounded the superior posterior branch of the bronchus to the right lower lobe. One hila gland was enlarged by white growth. No other secondary deposits seen.

The cerebral arteries appeared normal. In the right occipital lobe, in the anterior-superior whit matter, there was a small thin zone of pale browi discoloration, apparently cerebral softening of some duration. The cerebellum, pons, medullia and upper cervical cord appeared normal.

\section{Histology}

The growth in the lung was a typical oat-cel carcinoma. In the central nervous system the most marked changes were found in the cere bellum. Many of the Purkinje cells had coms pletely disappeared and survivors were roundeg and contracted with loss of the dendritic processes (Fig. I). The associated basket cells appeared more numerous. In the molecular layer prolifera? tion of microglial cells was a marked feature and well shown by Hortega's silver carbonate stain (Fig. 2). These changes in the cerebellar cortex were found to be equally marked in the laterap 


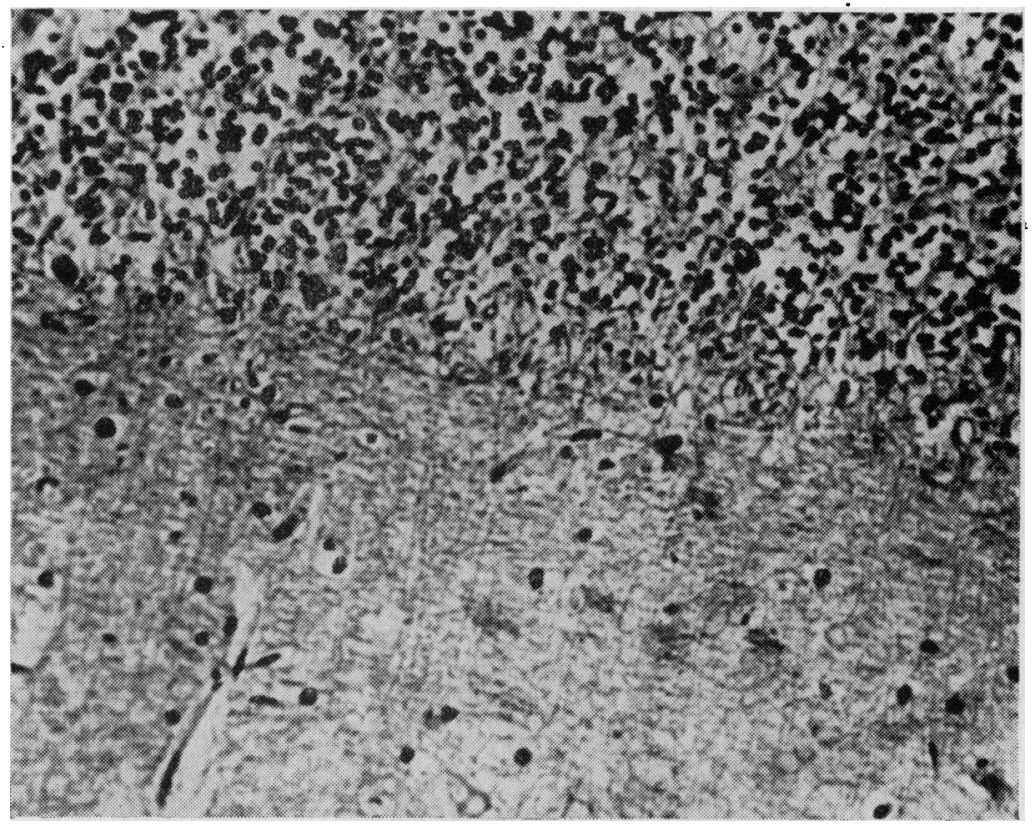

Fig. 1.-Cerebellar cortex showing diminution of number of Purkinje cells. H. and E. $\times 240$.

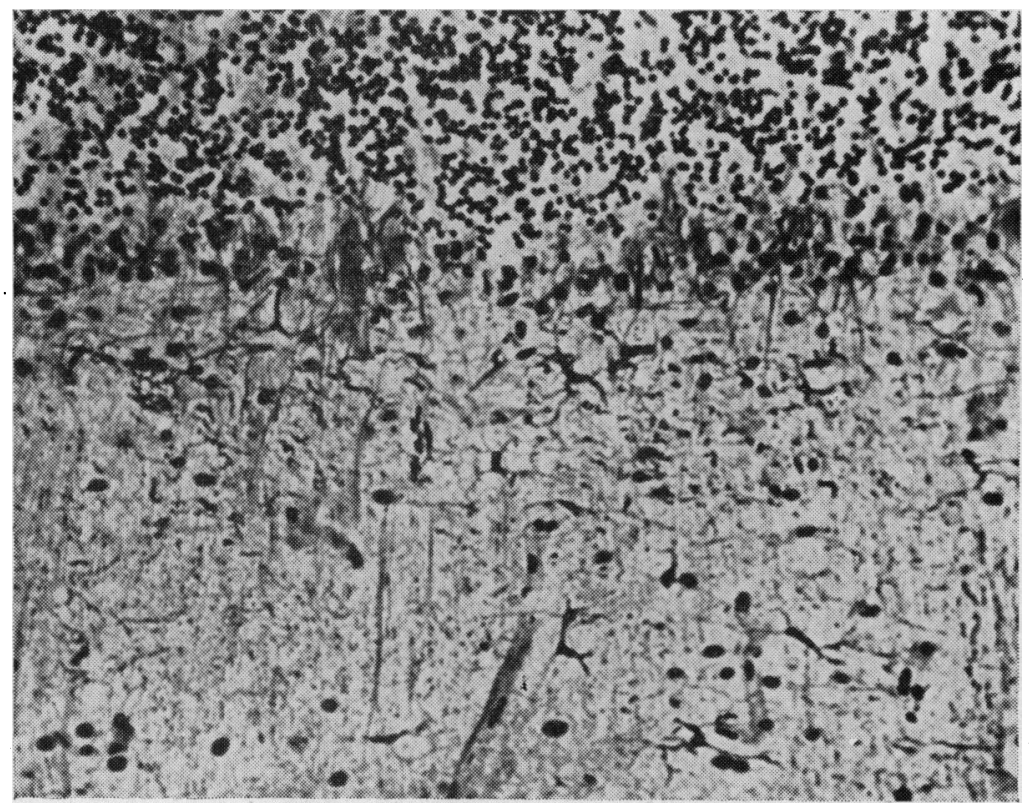

Fig. 2.- Junction of molecular and granular layers of cerebellar cortex showing numerous microglial cells with branching processes in the molecular layer. Hortega's silver carbonate $\times 240$.

lobes and in the vermis. In the white matter of the cerebellum adjacent to the dentate nucleus severe demyelinization had occurred and swollen glial cells laden with lipoid were present in large numbers (Fig. 3). The neurones of the dentate nucleus were degenerate and contracted in the 


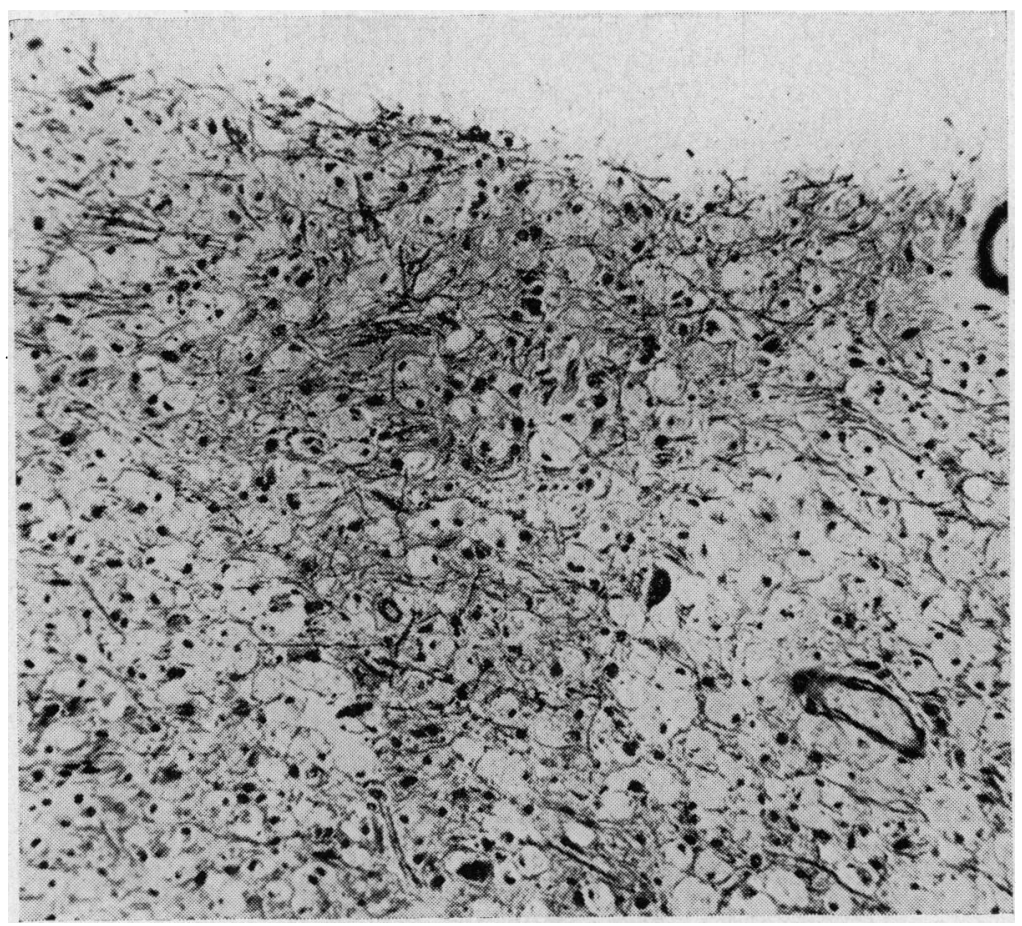

FIG. 3.-White matter of cerebellum stowing area of demyelinization with marked accumulation of large lipoid laden cerebral histiocytes. H. and E. $\times 270$.

regions contiguous to the area of demyelinization. Occasionally, small vessels showed perivascular lymphocytic infiltration. Sections of the pons and medulla were normal. Upper cervical cord sections showed no evidence of tract degeneration. A section of the focus of softening seen in the right occipital lobe showed this to be of typical ischæmic type with numerous compound granular corpuscles and some iron-containing phagocytes, with a surrounding area of astrocytic gliosis.

\section{Discussion}

Lennox and Prichard (1950) demonstrated that the association between bronchial carcinoma and neuropathy was statistically significant. By $195^{8}$, Brain and Henson had collected 42 carcinomatous neuropathies in 32 of which the carcinoma arose in the bronchus. It was stressed by Henson, Russell and Wilkinson (1954) that there was no constant time relationship between the onset of the neurological syndrome and the discovery of the underlying neoplasm. Either condition may appear first, months or even years elapsing before the other manifests itself. Henson, Russell and Wilkinson (1954) quote Ironside's case in which sensory neurnpathy preceded the discovery of a bronchial carcinoma by $3 \frac{1}{2}$ years. In addition there appears to be no correlation between the size of tumour and severity of the neuropathy Surgical removal of the tumour has no benefician effect on an already established neurological lesior $\vec{b}$ indeed the neuropathy may first arise after ex cision of the carcinoma. Heathfield and Williams (1954) noted that of ${ }_{15}$ cases of carcinomatous neuropathy, in which the histology of the bronchiaf carcinoma is recorded, I I are described as oat ce $\bar{P}$. in type, three as squamous, and one as undifo ferentiated.

The present case of subacute cerebellar degener ation associated with bronchial carcinoma is an example of one of the less common neuropathies Greenfield (1934) described two cases of 'subacute spino-cerebellar degeneration ', one of which haf an associated carcinoma of the bronchus. In this' case long tract degeneration was present in addis tion to loss of Purkinje cells and degeneration of Luysian nuclei. Greenfield at that time did not suspect an association between the two conditions Brain, Daniel and Greenfield (I95I) reportef four cases of subacute cortical cerebellar degenerạ tion in three of which there was also degeneration of the long tracts of the spinal cord. In these्ब 
three cases, two had a carcinoma of the bronchus and one an ovarian carcinoma. On reviewing the literature they collected 16 cases of subacute cortical cerebellar degeneration, of which i I had an underlying neoplasm (five ovary, three bronchus, two uterus and one breast). Henson, Russell and Wilkinson (1954) described in a series of 19 patients with carcinomatous neuropathy and myopathy, five in whom cerebellar symptoms and signs predominated. The usual clinical picture has been one of progressive cerebellar ataxia, nystagmus and dysarthria. Other features often encountered are diplopia, euphoria and dementia, pains in the limbs, sensory disturbances, deafness, atrophic paresis and pyramidal signs (Henson, 1953). In some of these patients the cerebrospinal fluid has shown an increased cell and protein content with a paretic Lange curve.

The manner of presentation in our case bears strong resemblance to the above clinical description. The very rapid cerebellar deterioration during the first few weeks of her eight months' illness follows closely the pattern described by Brain, Daniel and Greenfield (195I) and Henson, Russell and Wilkinson (1954). It was particularly interesting to note the severe mental deterioration of our patient, which has been well documented in other accounts. Rigorous search for an underlying tumour should be undertaken in all cases of a similar cerebellar neuropathy. In our patient three $\mathrm{X}$-rays of the chest were negative. The histological findings in the cerebellum in our case agree closely with the observations by Greenfield (1954), the degeneration being diffuse with no tendency to special involvement of the neo- or pallio-cerebellum. The disappearance of Purkinje cells with almost no damage to the basket cells is most striking. The involvement of the dentate nucleus is also a prominent feature. As mentioned previously, spinal cord tract lesions may occur, but, unfortunately, only the upper cord in our patient was available for histology, and this showed no abnormality.

The atiology of this interesting neurological syndrome is not known, but most workers seem agreed that some common factor probably accounts for both conditions, i.e. that the carcinogen may cause both the neuropathy and the carcinoma. If the neurological picture was produced by toxins from the carcinoma, one would expect to find a correlation with the size of the tumour, which does not occur. Other theories which have been advanced include virus infection, sensitization, and possible metabolic disorder.

\section{Summary}

A case of subacute cerebellar degeneration associated with carcinoma of the bronchus is described. Post-mortem confirmation was obtained. The literature is briefly reviewed.

We wish to thank Dr. J. Davson for the post-mortem report and Miss J. L. Perry of the Photographic Department for the microphotographs.

\section{REFERENCES}

Brian, W. R., Daniel, P. M., and Greenfield, J. G. (1951): Subacute Cortical Cerebellar Degeneration and its Relation to Carcinoma, $\mathcal{F}$. Neurol. Psychiat., 14, 59.

- and Henson, R. A. (1958): Neurological Syndromes Associated with Carcinoma, Lancet, ii, 97 I.

Greenfield, J. G. (1934): Subacute Spino-Cerebellar Degeneration Occurring in Elderly Patients, Brain, 57, r6r.

- (1954): The Spino-Cerebellar Degenerations, rst edition. Oxford: Blackwell.

Heathrield, K. W. G., and Williams, J. R. B. (1954): Peripheral Neuropathy and Myopathy Associated with Bronchogenic Carcinoma, Brain, 77, 122.

Henson, R. A. (1953): Discussion on Unusual Manifestations of Bronchial Carcinoma, Proc. Roy. soc. Med., 46, 859. , Russell, D. S., and Wilkinson, M. I. P. (1954): Carcinomatous Neuropathy and Myopathy-a Clinical and Pathological Study, Brain, 77, 82.

Lennox, B., and Prichard, S. (1950): The Association of Bronchial Carcinoma and Peripheral Neuritis, Quart. $\mathcal{J}$. Med., r9, 97. 